\title{
Evaluating a knowledge exchange intervention in cancer survivorship care: a workshop to foster implementation of Online Support Groups
}

\author{
Arminee Kazanjian • Kirsten Smillie • Joanne Stephen
}

Received: 22 August 2012 /Accepted: 10 December 2012 /Published online: 29 December 2012

(C) The Author(s) 2012. This article is published with open access at Springerlink.com

\begin{abstract}
Purpose The purpose of the research described here is to assess the overall effectiveness of the workshop format as a Knowledge Exchange (KE) strategy in (1) disseminating scientific evidence, clinical experience, and systems information related to professionally led Online Support Groups (OSG) for cancer survivors and (2) facilitating the implementation of this intervention by a select group of end users - decision makers and clinical leads in psychosocial supportive care.

Methods The KE-Decision Support (KE-DS) Model, operationalizing the Health Technology Approach, guided the development of pre- and postworkshop questionnaires, and a follow-up questionnaire administered 5 months after the workshop. Questionnaire results were categorized according to participants' responses to these elements: methods of engagement, evidence (scientific, experiential, systems) and the delivery of this evidence, and external factors at the institutional level, such as administrative support, budgetary issues, etc., that influence decision-maker abilities and strategies.
\end{abstract}

Electronic supplementary material The online version of this article (doi:10.1007/s00520-012-1686-2) contains supplementary material, which is available to authorized users.

A. Kazanjian $\cdot$ K. Smillie $(\bowtie)$

School of Population and Public Health, University of British Columbia, Vancouver, BC, Canada

e-mail: kirsten.smillie@ubc.ca

A. Kazanjian

e-mail: arminee.kazanjian@ubc.ca

J. Stephen

Fraser Valley Centre, BC Cancer Agency,

Vancouver, BC, Canada

e-mail: jstephen@bccancer.bc.ca
Results Traditional KE strategies such as peer-reviewed journal articles are optimal for disseminating scientific evidence, while face-to-face interactions, such as in a workshop, are best used to disseminate systems-level implementation information, such as fiscal implications, budgetary requirements, and policy relevance, which is not found in journal articles or conferences. An apparent shift in workplace culture signifies the availability of institutional support for high-level staff to engage in KE.

Conclusions As a KE strategy with identified end users, the workshop format is effective in facilitating the implementation of this intervention in participants' institutions.

Keywords Knowledge exchange $\cdot$ KE evaluation · Supportive cancer care
Abbreviations
KE Knowledge exchange
OSG Online Support Groups

\section{Background}

Current state of KE literature/science

The timely exchange of information and evidence is recognized by researchers, clinicians, and decision makers alike as critical to promoting best practices in health care [1-3]. Despite increased awareness of its importance, evidence-informed decision making has yet to be fully realized, and the challenges associated with this exchange have received significant attention in the literature [4-6]. Knowledge exchange (KE) is the most commonly used term to describe this process. While a 
plethora of definitions exist, some are more comprehensive than others [7]. A number of strategies to facilitate KE between knowledge creators and users have emerged. For example, funding organizations in Canada regularly require researchers to plan and implement $\mathrm{KE}$ strategies in their programs of research, and increasingly, the importance of assessing the implementation and impact of research in a policy environment has been recognized $[6,8]$. While substantial work has gone into developing $\mathrm{KE}$ strategies, there remain significant barriers to knowledge uptake, including time, culture, resources, and incentives [9]. Moreover, systematic approaches targeting administrators and policy makers to facilitate KE continue to be underdeveloped and underexamined.

Regardless of the increased attention to bridging the "knowdo gap" [10], a paucity around primary research on KE itself remains - that is, what KE strategies are the most effective in which contexts [11] and the scientific underpinning of KE, for example, the effectiveness of clinical guidelines [12]. Much of the current $\mathrm{KE}$ literature is predominately descriptive in nature, considering aspects such as barriers and facilitators to effective $\mathrm{KE}$ strategies. This is an important area of research and discussion, and our own previous work contributes to it [13]. KE by its nature is an inherently nonlinear and complex processunderstanding a KE strategy in one context does not necessarily translate to understanding it in another [14]. Through evaluation, however, a better understanding of end users' decisionmaking context may result, and KE strategies may be refined and tailored to better meet this need [15].

\section{The Knowledge Exchange-Decision Support Model}

\section{The Knowledge Exchange-Decision Support (KE-DS) Model} is a relational, structured approach to KE [16]. Expanding and further operationalizing the conceptual framework of Health Technology Assessment, the model is designed to delineate the iterative set of interactions between the creation of new information, strategies for dissemination and translation, and activities related to implementation of new knowledge into current practice. The KE-DS Model reflects the principles of evidence-informed practice. Advocates posit that researchers translate results and evidence, usually expressed from a disciplinary perspective, into a meaningful product for knowledge users, and that knowledge users inform the research agenda so that, in turn, meaningful questions and issues are investigated. Dialog and interaction at all stages of the research process are reported to be key $[17,18]$.

The KE-DS Model's distinguishing feature is its explicit examination of individual, organizational, and systemic levels to best support KE behavior and action. In this context, the local conditions and setting are equally as important to the success of the program or intervention as the effectiveness evidence of the program itself. The model is ideal for those making complex decisions at the program or policy level [19] that "do not fit into neat little boxes that can be informed by technically oriented inputs" [20]. See Kazanjian et al. (2010) for a detailed description of the KE-DS Model and Kazanjian et al. (2011) for its application to the implementation of a supportive cancer care program [13, 14].

While our model is comprehensive in its approach to KE, the natural experiment reported here focuses on a specific stage of the KE process - the evaluation of knowledge dissemination and consequent program implementation. Our aim is to gain a better understanding of the effectiveness of the workshop format as a KE strategy. As part of a panCanadian program of research in cancer supportive care, the KE-DS team partnered with the Online Support Group (OSG) advisory committee to evaluate a workshop aimed at educating potential supporters about the program and securing their commitment to deliver and fund OSG in their respective regions.

\section{Methods}

The purpose of this study is to assess the overall effectiveness of the workshop format as a KE strategy in:

1. Disseminating scientific evidence, clinical experience, and systems information related to OSG and

2. Facilitating the implementation of this intervention by a selected group of end users (decision makers and clinical leads)

\section{Description of program — Online Support Groups}

Professionally led online group support is an emerging area in supportive cancer care with little precedent. Through a 4year pilot study (2007-2011), Stephen et al. illustrated the intervention's value in meeting the needs of cancer survivors who face barriers to accessing supportive cancer care, such as geographic barriers, financial resource constraints, and a fragmentation of services between provincial care centers and health authorities [21]. There remain challenges to establishing OSG as a sustainable and accepted service. A literature review and environmental scan conducted by the OSG research team revealed prevalent concerns and negative attitudes within the clinician and program leadership community regarding online interventions, such as the clinicianpatient relationship, patient safety and privacy issues, data security and risk management issues, and unclear policy around jurisdictional issues. There was broad variation in the attitudes reported in current literature on psycho-technologies $[22,23]$, including the view that as patients are already seeking and accessing psycho-technologies, online services delivered by professionals who develop and maintain expertise are the most likely to be safe and effective. 


\section{Description of workshop}

In response to these issues, the OSG advisory committee developed procedures and protocols to address the ethical/ risk management issues and the activities and role functions needed for implementation. A workshop was identified as an optimal strategy to facilitate the exchange of knowledge from the pilot project with those in supportive cancer care. Clinical leads and decision makers in the area of supportive cancer care were identified as the ideal participants for this stage of stakeholder outreach. The workshop would allow the OSG advisory committee the opportunity to present evidence and information related to OSG implementation and workshop participants the opportunity to ask questions and clarify uncertainties related to OSG. Furthermore, participants would be given the opportunity to express their interest in partnering with the OSG advisory committee to deliver and fund OSG in their regions.

Aside from two researchers (AK, KS) and two OSG staff, 29 people attended the 1-day workshop: 6 members of the advisory committee, 5 clinicians, 14 decision makers, and 4 clinical program coordinators. The advisory group was composed of clinician-researchers who had been previously identified on the basis of two criteria: relevant clinical expertise and pan-Canadian representation. The remaining participants at the workshop were decision makers or delegates of decision makers who represented supportive care cancer center programs across Canada. Invitees were selected on the basis of the cancer center they represented: the intent was to have pan-Canadian representation from cancer centers that served a significant nonurban population. Fewer than half of the invitees were known to members of the advisory group or each other. The workshop involved a presentation by a member from the national organization funding the OSG pilot work, a presentation of the current research evidence by the principal investigator (coauthor JS), a demonstration of the online platform that OSG operates from, ${ }^{1}$ a small group discussion around implementation in specific regions, and a larger group discussion on barriers and opportunities to implementing OSG nationally. The workshop took place in Canada in February 2011.

\section{Data collection and analysis for workshop evaluation}

The KE-DS Model guided the development of pre- and postworkshop surveys (see Additional files 1 and 2), and a follow-up survey (see Additional file 3) administered 5 months after the workshop (Table 1). The 5-month follow-up questionnaire was administered through Survey Secure, an online survey service that was emailed to

\footnotetext{
${ }^{1}$ Cancer Chat Canada is the website and online platform through which online support groups are offered: http://cancerchatcanada.ca/.
}

participants. They received a reminder email 3 weeks after and a final reminder with the survey attached as a word document 7 weeks later. Our evaluation of the workshop incorporates all related activities, from the initial invitation to the final postworkshop follow-up at 5 months. Through analysis of the pre- and postworkshop questionnaires and notes taken by two of the coauthors (AK, KS) during the workshop, the KE-DS research team identified variables, or themes, that warranted further exploration in the follow-up questionnaire, particularly around the influence of external institutional elements (i.e., policies and procedures, funding, etc.) they would have encountered after the workshop once they returned to their jurisdictions. Using the KE-DS Model as analytic framework, we examined survey responses for key aspects in information seeking and retrieval, interpretation, communication, and contextualization. We further explored reported expressions of knowledge to action issues to identify current gaps in understanding that step.

\section{Results}

Twenty three of the 29 eligible participants completed the preworkshop questionnaire $(n=23), 24$ completed the postworkshop questionnaire $(n=24)$, and 13 completed the follow-up questionnaire $(n=13)$. Participants were asked to check all titles that applied to their role. Of the 23 who responded, 12 identified as clinicians, 11 as clinical leaders, 13 as administrator/managers, 9 as researchers, and 3 as "other." Participants were asked to describe their knowledge of the research evidence related to OSG before the workshop. Two out of 23 participants self-identified as expert, 8/23 as knowledgeable, 7/23 as somewhat knowledgeable, and 7/23 as having limited knowledge.

Results are discussed according to participants' responses to these dimensions:

- Methods of engagement (emails, slide show, journal articles, small group work, workshop as a whole)

- Evidence (scientific, experiential, systems) and the delivery of this evidence

- Factors at the institutional level, such as administrative support, culture of workplace, budgetary issues, new programming policies, etc., that differentially influence decision-making abilities and strategies

Methods of stakeholder engagement

Prior to the workshop, a series of emails were sent by the OSG advisory committee to invitees that contained a slideshow and two manuscripts. In the postworkshop questionnaire, all participants said that receiving the emails containing the supplemental material prior to the workshop prepared them 
Table 1 Questionnaires administered pre and postworkshop, and 5 months following the workshop

\begin{tabular}{|c|c|c|c|}
\hline Questionnaire & Sample size & Time administered & Focus \\
\hline $\begin{array}{l}\text { Preworkshop } \\
\text { questionnaire }\end{array}$ & $n=23$ & Morning of the workshop & $\begin{array}{l}\text {-Professional role } \\
\text {-Preferred methods of engagement } \\
\text { - Types of evidence consulted in their professional roles } \\
\text { - Current KE activities } \\
\text {-Level of knowledge of OSG } \\
\text { - Helpfulness of preworkshop material sent out via email }\end{array}$ \\
\hline $\begin{array}{l}\text { Postworkshop } \\
\text { questionnaire }\end{array}$ & $n=24$ & $\begin{array}{l}\text { At the end of workshop } \\
\text { on the same day }\end{array}$ & $\begin{array}{l}\text {-Effectiveness of the workshop in increasing their knowledge of OSG } \\
\text {-Relevancy of workshop content to their professional roles } \\
\text {-If OSG is an intervention that would be supported in their region }\end{array}$ \\
\hline $\begin{array}{l}\text { Follow-up } \\
\text { questionnaire }\end{array}$ & $n=13$ & 5 months after the workshop & $\begin{array}{l}\text {-Institutional elements (i.e., policies, availability of support, budget, values, etc.) } \\
\text { encountered in their institution after the workshop }\end{array}$ \\
\hline
\end{tabular}

$(24 / 24)$, and the majority stated that there was no other information that would have better prepared them for the meeting (20/24). The large majority of participants read the two manuscripts (19/23 and 17/23) and found them most useful in providing background information about OSG and disseminating research evidence. Only half of participants (12/23) who completed the preworkshop questionnaire watched the slideshow.

All respondents who completed the postworkshop questionnaire $(n=24)$ stated that the workshop was relevant to their work in supportive care, that the material was appropriate for those invited, that the focus was what they expected, and that their questions and concerns were addressed. The workshop was most effective in translating the current activity of the National Steering Group, with the vast majority (18/24) reporting it to be very effective. Just under half of participants $(10 / 24)$ reported the workshop to be very effective in increasing their knowledge of the research evidence related to OSG. When asked to identify all that they found beneficial about the workshop, most respondents indicated the opportunity to communicate matters of strategy and practical issues related to implementation with others in supportive care $(21 / 24)$ and create an action plan for how to implement OSG in their area (17/24).

The value of evidence: scientific and other

In the preworkshop questionnaire, participants were asked to identify all of the methods they used to stay abreast of current research in relation to supportive cancer care. Publications were the most highly reported method (17/23), followed by conferences (12/23), colleagues/word of mouth $(12 / 23)$, and continuing education sessions (11/23).

In the 5-month follow-up survey, all respondents $(n=13)$ said that they had the opportunity to discuss OSG with their colleagues and that they had a good grasp of what OSG are about. All but one said they felt that they could convey this knowledge to others they work with and that they returned to their work place after the workshop with the intent of finding a way to implement OSG in their region (12/13 participants). Participants were asked which strategies would be useful in making the most of existing supports for OSG in their region/ institution. Most participants reported the usefulness of a detailed outline of budgetary requirements to implement OSG (11/13), followed by published peer-reviewed journal articles (7/13). If the aim is to tackle barriers related to implementing OSG, participants identified a detailed outline of budgetary requirements to be the most helpful (8/13), followed by a short research brief summarizing findings into policy and program relevant messages (6/13).

Institutional-level factors that influence decision making

In the preworkshop questionnaire $(n=23)$, participants were asked about the types of supports they were offered to access research evidence in their everyday practice. Nearly all participants $(22 / 23)$ said that they received support. Time off work to attend conferences, presentations, rounds, etc. were the most common form of reported support (21/23), followed by research assistance through an individual such as a librarian or educator (20/23) and encouragement to participate on research teams $(18 / 23)$.

In the postworkshop survey $(n=24)$, participants were asked if OSG are an intervention that would be supported by their institution. Nearly all respondents (20/24) said yes, and 5/24 said unsure (two people checked both yes and unsure). The majority of participants identified budgetary restrictions as an issue (14/24), followed by limited professional capacity to follow through $(8 / 24)$ and uncertainty regarding effectiveness of OSG (3/24). Comments included "budgetary restrictions could be problematic"; "limited resources, program planning"; and "cost/benefit analysis need to be conducted to build business case for long term clinical secure viability." 
In the follow-up survey 5 months after the workshop $(n=13)$, participants were asked about current supports in their region/at their institution that would assist in implementing OSG, a commitment to support cancer care programming was the most common (12/13), followed by a need for services in rural areas (11/13) and existing moral support from colleagues and clinicians (10/13). Participants were asked to identify all the reasons why OSG are not/may not be right for their jurisdiction. Again budgetary issues were the most frequently cited: $4 / 13$ said costing implications for operating OSG are not clear; another 3/13 said uncertainty with funding a service that crosses provincial jurisdictions.

\section{Discussion}

The goal of the OSG advisory committee was to educate workshop participants about the of OSG program (disseminate) with the specific objectives of presenting information relevant to previously identified barriers, specifically, clinical/relational aspects of the therapeutic intervention and organizational challenges and logistics. The information presented and the facilitated discussion and brainstorming sessions were designed to advance decision makers from contemplation to commitment to deliver and fund an OSG program in their regions (implementation). Of the 14 decision makers who attended the workshop, 13 committed to partnering with the OSG national advisory committee to offer OSG in their regions and dedicate some form of resource.

According to questionnaire results, the dissemination of information at the workshop (including emails sent before) was successful. The opportunity to network and converse with others face to face was identified as the most beneficial thing about this KE strategy, a point substantiated in the KE literature [24-26]. Interestingly, learning about OSG was the least identified benefit of the workshop, and less than half of responders classified it as "very effective" in increasing their knowledge of the research evidence related to OSG. This does not suggest that the workshop failed in educating participants about OSG; rather, this high-level group of research users regards scientific evidence as but one type of information relevant to their decision-making roles. This group of high-level end users appears to utilize traditional methods of KE, such as peer-reviewed articles, through which to stay current of scientific evidence. They are well supported to seek scientific evidence at the institutional level, a key factor also identified in the KE literature $[27,28]$. Face-to-face meetings, therefore, may be regarded as an activity better suited to communicating with others about the logistical and strategic aspects of implementation, rather than to learn about and understand the scientific evidence. While conversing and networking was not a primary goal of the workshop, the workshop accomplished the two other goals of dissemination and garnering commitment for implementation. When asked to identify the most significant topic covered, responses included information to move forward, the sustainability and feasibility of OSG, and a variety of region/province-specific discussions:

- "Moving forward to my region, continuing conversations and building support and partnerships"

- "Sustainability of OSG"

- "Necessary training to provide the type of care, next steps for sustainability"

- "Discussion in small groups within provinces"

- "Clear demonstration of feasibility and safety of OSG"

- "Practical issues; how to move on"

As these individuals come from a diversity of institutions, the fact that nearly all of them are well supported in accessing and consulting the research evidence could be due to selection bias or may suggest a cultural shift where the importance of evidence-informed decision making is not only recognized at the organizational level, but also facilitated. While much of the early literature supports the idea that face-to-face opportunities are often more effective than printed material in facilitating knowledge exchange $[9,29,30]$, for individuals who have research support, published material remains an optimal method of disseminating scientific evidence and, in so doing, frees up face-to-face meetings to focus on systems level or implementation information, such as pertinent policies, budgetary requirements, and technical issues.

In a review of the KE literature, "acceptable evidence for decision makers can be less rigorous than that for researchers and includes gray literature (i.e., government publications, consultants' reports, monographs and conference proceedings)" and that in one study, "decision makers persistently valued experience more than they did research" [20]. The evidence exchanged at the workshop included scientific (research evidence), experiential (that of OSG facilitators), and systems level (information related to uptake, implementation, and maintenance as defined by Best, 2009) [26]. Scientific evidence is clearly valued by this group - research briefs and peer-reviewed papers were identified as being helpful in conveying knowledge to others about OSG, in making the most of existing supports and to tackling barriers to implementing OSG. At the same time, however, discussion of the scientific evidence did not dominate the workshop. The initial concerns by the OSG advisory committee that the risks and effectiveness of OSG were not clearly understood by clinicians did not appear to be an issue for those who completed the follow-up questionnaire. Only one person identified "uncertainty regarding patient privacy and data security" and "uncertainty regarding effectiveness of OSG" as reasons for why OSG may not be right for their region. The main concern by this group was related to an absence of precedent for a national approach and practical details such as cost sharing 
formulas and professional accreditation. Lasting concerns seem to be related to logistic and administrative issues rather than with OSG as a method of psychosocial counseling.

Analysis indicates that the implementation of the information exchanged at the workshop was successful. Since the workshop, 13 of the 14 decision makers have committed resources from their cancer center-either their own time in the leadership group and/or clinical staff time for facilitation, to the OSG initiative. For those who stated that OSG is not right for their institution, more people said that this was due to uncertainty around budgetary implications rather than simply having budgetary restrictions. This decision appears to be influenced by the lack of funding information rather than the lack of available funds, or disinterest, in the program.

A desire for systems-level information reflects the types of knowledge needed by decision makers in different jurisdictions. While decision makers are responsible for identifying a program or intervention that is evidence based, they must also illustrate how this evidence will translate in their local, institutional setting [26]. Systems-level information is not likely to be found in the scientific literature, nor is it common in health services research and policy reports, despite a call by many for this to change [25, 31]. While this workshop was successful in disseminating information related to OSG, there was a need for more systems-level information by some participants.

This small-scale study has two limitations. First, due to the number of participants, the generalizability of findings is limited. Replication of findings through several more case studies would address this limitation. Second, selection bias may affect the measures of KE success as participants were selected by the OSG advisory committee, they may already have been informed of the evidence related to OSG, and rather predisposed to commit to funding and offering OSG through their institutions regardless of the workshop. However, since there is still appreciable variation in the level of commitment, selection bias alone could not explain these results.

\section{Conclusions}

KE is a complex and multifaceted process that cannot easily be replicated from one context to the next [32]. Evaluation of specific strategies, however, could lead to both a better understanding of the types of information that clinicians and decision makers need and the most effective communication approaches through which to engage them [33]. The KE-DS Model offers a comprehensive approach to mapping knowledge exchange, thus making transparent the potential opportunities for knowledge transfer, as well as the evaluation of specific dissemination and implementation strategies.
OSG is a relatively new intervention that has been proven effective through early scientific research. As there is little precedent for this type of professionally led online service, implementation information around policies, budget, and professional accreditation is limited. Through the evaluation of a workshop with invited decision makers and clinical leads, the advisory committee learned that this group of end users desired systems-level information related to implementation. A face-to-face meeting is best suited to facilitate networking and disseminate implementation and policy relevant information. Peer-reviewed journal articles and conferences remain optimal methods of dissemination for research evidence. This group of end users is well supported at the organizational level to stay current of scientific evidence, marking a shift in health care culture where the importance of KE is recognized and encouraged. This workshop was effective in both disseminating information through emails sent with published, peerreviewed journal articles attached and facilitating the implementation of this evidence by securing 13 participants' commitment to dedicate resources towards OSG. Evaluation of $\mathrm{KE}$ activities is an important component of facilitating evidence-informed decision making and will lead to improved understanding of these complex processes.

Acknowledgments This study was made possible by funding from the Canadian Institute of Health Research (\#AQC83559) and the Canadian Partnership Against Cancer.

Conflict of interest None.

Open Access This article is distributed under the terms of the Creative Commons Attribution Noncommercial License which permits any noncommercial use, distribution, and reproduction in any medium, provided the original author(s) and the source are credited.

\section{References}

1. Kelly AM (2009) Evidence-based practice: an introduction and overview. Semin Roentgenol 44(3):131-139

2. Kerner J, Rimer B, Emmons K (2005) Introduction to the special section on dissemination: dissemination research and research dissemination: how can we close the gap? Heal Psychol 24(5):443-446

3. Oxman AD, Fretheim A, Schunemann HJ (2006) SURE: improving the use of research evidence in guideline development: introduction. Health Res Policy Syst 4:12

4. Graham I, Logan J, Harrison M, Straus S, Tetroe J, Caswell W et al (2006) Lost in knowledge translation: time for a map? J Contin Educ Heal Prof 26:13-24

5. Hennink M, Stephenson R (2005) Using research to inform health policy: barriers and strengths in developing countries. J Heal Commun 10:163-180

6. Lavis J, Robertson J, Woodside C, McLeod C, Abelson A (2003) How can research organizations more effectively transfer research knowledge to decision makers? Milbank Q 81:221-248

7. Canadian Health Services Research Foundation (2012) Knowledge exchange [http://www.cihr-irsc.gc.ca/e/39033.html]. Accessed on December 17, 2012 
8. Lavis J, Ross S, Hurley J, Hohenadel J, Stoddart G, Woodward C, Abelson J (2002) Examining the role of health services research in policy making. Milbank Q 80(1):125-154

9. Lomas J (2000) Connecting research and policy. ISUMA: Can J Policy Res 1(1):140-144

10. World Health Organization (2006) Bridging the "know-do" gap meeting on knowledge translation in global health. 10-12 October, 2005 World Health Organization, Geneva, Switzerland. [http:// www.who.int/kms/WHO_EIP_KMS_2006_2.pdf]. Accessed on March 5, 2012

11. Mitton C, Adair C, McKenzie E, Patten S, Perry BW, Smith N (2009) Designing a knowledge transfer and exchange strategy for the Alberta Depression Initiative: contributions of qualitative research with key stakeholders. Int J Ment Heal Syst 3:11

12. Brouwers MC, Garcia K, Makarski J, Daraz L, for the Evidence Expert Panel of the KT for Cancer Control in Canada Project Research Team (2011) The landscape of knowledge translation interventions in cancer control: what do we know and where to next? A review of systematic reviews. Implement Sci 6:130

13. Kazanjian A, Smillie K, Howard AF, Ward A, Doll R (2012) A structured approach to knowledge exchange: understanding the implementation of a cancer survivor program. Eur J Oncol Nurs 16:399-405

14. Kazanjian A, Chan V, Smillie K, Howard F (2010) Knowledge exchange decision support (KE-DS) toolkit. http://spph.ubc.ca/ sites/healthcare/files/2010/KEDS_Toolkit_Nov2010.pdf. Accessed on August 20, 2012

15. Grimshaw J, Eccles M, Lavis J, Hill S, Squires J (2012) Knowledge translation of research findings. Implement Sci 7(50)

16. Best A, Hiatt R, Norman C (2008) Knowledge integration: conceptualizing communications in cancer control systems. Patient Educ Couns 71:319-327

17. Lavis J (2006) Research, public policymaking, and knowledgetranslation processes: Canadian efforts to build bridges. J Contin Educ Heal Prof 26:37-45

18. Ross S, Lavis J, Rodriguez C, Woodside J, Denis J (2003) Partnership experiences: involving decision makers in the research process. J Health Serv Res Policy 8:26-34

19. Pawson R, Greenhalgh T, Harvey G, Walshe K (2005) Realist review - a new method of systematic review designed for complex policy interventions. J Health Serv Res Policy 10(1):21-34
20. Mitton C, Adair C, McKenzie E, Patten S, Perry BW (2007) Knowledge transfer and exchange: review and synthesis of the literature. Milbank Q 85(4):729-768

21. Stephen J, Taylor Brown J, Christie G, Flood K, Golant M, Rennie H, Gordon S, Rahn M, Speca M, Turner J (2011) The learning experience of professional facilitators of online support groups for cancer survivors. Psychooncology 20:832-840

22. Caspar F, Berger T (2005) The future is bright: how can we optimize online counselling, and how can we know whether we have done so? Counselling Psychol 33:900-909

23. Mallen MJ, Vogel DL, Rochlen AB (2005) The practical aspects of online counselling: ethics, training, technology, and competency. Counselling Psychol 33(6):776-818

24. Hanney SR, Gonzalez-Block MA, Buxton MJ, Kogan M (2003) The utilisation of health research in policy-making: concepts, examples and methods of assessment. Health Res Policy Syst 1:2

25. Philip KL, Backettee-Milburn K, Cunningham-Burley S, Davis JB (2003) Practising what we preach? A practical approach to bringing research, policy and practice together in relation to children and health inequalities. Heal Educ Res 18(5):568-579

26. Best A, Terpstra JL, Moor G, Riley B, Norman CD, Glasgow RE (2009) Building knowledge integration systems for evidenceinformed decisions. Knowl Integr Syst 23(6)

27. Bowen S, Martens P, the Manitoba Need to Know Team (2005) Demystifying knowledge translation: learning from the community. J Health Serv Res Policy 10(4):203-211

28. Rosenheck RA (2001) Organizational process: a missing link between research and practice. Psychiatr Serv 52:1607-1612

29. Roos N, Shapiro E (1999) From research to policy: what have we learned? Med Care 37(6):JS291-JS305

30. Soumerai S, Avorn J (1990) Principles of educational outreach ("academic detailing") to improve clinical decision-making. JAMA 263:549-556

31. Glasgow R, Green L, Klesges L, Abrams D, Fisher E, Goldstein M, Hayman L, Ockene J, Orleans T (2006) External validity: we need to do more. Soc Behav Med 31(2):105-107

32. Contandriopoulos D, Denis JL, Tremblay E (2010) Knowledge exchange processes in organization and policy arenas: a narrative systematic review of the literature. Milbank Q 88(4):444-483

33. Greene SM, Reid RJ, Larson EB (2012) Implementing the learning health system: from concept to action. Ann Intern Med 157:207210 